\title{
On the theory of electron transfer reactions at semiconductor/liquid interfaces. II. A free electron model
}

\author{
Yi Qin Gao and R. A. Marcus \\ Noyes Laboratory of Chemical Physics, Mail Code 127-72, California Institute of Technology, Pasadena, \\ California 91125
}

(Received 10 March 2000; accepted 24 July 2000)

\begin{abstract}
Electron transfer reactions at semiconductor/liquid interfaces are studied using the Fermi Golden rule and a free electron model for the semiconductor and the redox molecule. Bardeen's method is adapted to calculate the coupling matrix element between the molecular and semiconductor electronic states where the effective electron mass in the semiconductor need not equal the actual electron mass. The calculated maximum electron transfer rate constants are compared with the experimental results as well as with the theoretical results obtained in Part I using tight-binding calculations. The results, which are analytic for an $s$-electron in the redox agent and reduced to a quadrature for $p_{z^{-}}$and $d_{z^{2}}$-electrons, add to the insight of the earlier calculations. (C) 2000 American Institute of Physics. [S0021-9606(00)70739-2]
\end{abstract}

\section{INTRODUCTION}

The electron transfer reactions at the $\mathrm{Si} / \mathrm{viologen}^{2+/+}$ and $\mathrm{InP} / \mathrm{Me}_{2} \mathrm{Fc}^{+/ 0}$ interfaces were studied recently by Lewis and co-workers. ${ }^{1-3}$ The experiments yielded a maximum electron transfer rate constant in the range of $10^{-17}-10^{-16} \mathrm{~cm}^{4} \mathrm{~s}^{-1}$. To compare with the experimental results, the maximum rate of interfacial electron transfer reactions between a redox agent in solution and InP and Si semiconductors was calculated in Part I, the InP surface, as is believed, being terminated with O's and the Si surface with H's. ${ }^{4}$ A tight-binding model was used for the semiconductor and extended Hückel calculations were performed for the molecule and for the electronic coupling, in conjunction with $z$-transform ${ }^{5}$ and slab methods. ${ }^{6}$ Since a free electron model for the problem provides a simple description which can add to the physical insight, the present treatment was undertaken, by adapting Bardeen's method to this study. It is known that with an effective mass the free electron model describes many properties of bulk semiconductors ${ }^{7-12}$ and that the free electron model with the actual electron mass describes various properties associated with the LCAO molecular wave functions of aromatics and polyenes, such as electron densities and bond orders ${ }^{13}$ (and so even the coefficients).

The paper is organized as follows: The theoretical model is given in Sec. II. The expression for the electron transfer rate constant and its application is given in Sec. III, and the results are compared with those in Part I and are discussed in Sec. IV.

\section{THEORY}

\section{A. Preliminary remarks}

The electrons in the semiconductor are treated here as free electrons in a semi-infinite potential well with a constant potential inside the well and a known effective mass. The potential well has a surface normal to the $z$ direction and is infinite in extent in the $x$ and $y$ directions. The electronic wave function of the molecule is obtained by solving a Schrödinger equation whose potential is constant inside a spherical potential well and is zero outside. Analogous models for molecules were applied in earlier studies of the orientation effect on the electron transfer reactions by Siders et al. ${ }^{14,15}$

The wave functions for the semiconductor electrode and the molecule obtained using the free electron model are then used to calculate the electronic coupling matrix element, and from it the maximum electron transfer rate constant. The electron transfer between a semiconductor electronic state and the molecular state is treated as nonadiabatic and Fermi Golden Rule is applied, and the electronic coupling matrix element is calculated by adapting the method introduced by Bardeen. ${ }^{16}$ The application of Bardeen's method, with an adaptation to the present case where the effective electron mass in a semiconductor differs from the actual electron mass, provides an analytical or quadrature expression for the coupling matrix element between the semiconductor and the molecular state.

The total electron transfer current between the semiconductor and the molecule is obtained as the sum of the currents between each semiconductor electronic state and the molecular state. This procedure was discussed and applied earlier in Part I to the study of electron transfer reactions at semiconductor/liquid interfaces using a tight-binding model. ${ }^{4}$ It has also been used by various groups in the study of electron transfer reactions at metal ${ }^{17-20}$ and semiconductor surfaces. ${ }^{18,21}$

The formula for the maximum rate constant is then applied to two semiconductor/liquid interfaces ( $\mathrm{Si} /$ viologen $^{2+/+}$ and $\left.\mathrm{InP} / \mathrm{Me}_{2} \mathrm{Fc}^{+/ 0}\right)$. These two interfaces were studied experimentally by Lewis and co-workers, ${ }^{1-3}$ and conditions were obtained for the former and partly for the latter which satisfied ideal current vs applied potential behavior. In these studies, the current density $J_{f}$ due to electron transfer from the semiconductor to the molecule is proportional to both the 
concentration $[A]$ of the molecules in the liquid and the density of electrons $n_{s}$ at the semiconductor surface, ${ }^{2}$

$$
J_{f}=e k_{\mathrm{et}} n_{s}[A],
$$

where $e$ is the elementary charge and $k_{\text {et }}$ is the electron transfer rate constant. These studies also provided experimental values for the maximum rate constant, which were around $10^{-16} \mathrm{~cm}^{4} \mathrm{~s}^{-1}$, for the electron transfer reaction at the two interfaces. The free electron model used in the present study provides rate constants for the two systems in reasonable agreement with the results of tight-binding calculations and with the experimental maximum rate constants.

\section{B. Kinetics at semiconductor/liquid interfaces}

The net current density $J$ due to the electron transfer reaction at a semiconductor $(S) /$ liquid interface,

$$
A+e(S) \rightleftharpoons A^{-}+S,
$$

can be written as

$$
J=J_{f}-J_{r},
$$

where $J_{f}$ is the current density due to the electron transfer from the semiconductor to the molecule and $J_{r}$ is the current density corresponding to the reverse process. $J_{f}$ and $J_{r}$ depend on the concentration of $A$ and $A^{-}$, respectively, at the interface,

$$
\begin{aligned}
& J_{f}=e k_{f}[A], \\
& J_{r}=e k_{r}\left[A^{-}\right],
\end{aligned}
$$

where $k_{f}$ and $k_{r}$ are pseudo-first-order rate constants, and, from Eq. (1),

$$
k_{f}=n_{s} k_{\mathrm{et}} .
$$

In the following, we obtain an expression for $k_{f}$ using a standard result ${ }^{22}$ on electron transfer reactions: Under the weak coupling assumption, the rate constant $k_{f}^{\mathrm{s}}$ for the electron transfer from a single electronic state of the semiconductor described by a superscript $s$, which includes both the effect of electron tunneling or hole and "nuclear reorganization," can be expressed using the Fermi Golden Rule, ${ }^{22}$ for an electronic state to electronic state transition,

$$
k_{f}^{\mathrm{s}}=\frac{2 \pi}{\hbar}|V|^{2} \mathrm{FC},
$$

where FC is the Franck-Condon factor, $V$ is the electronic coupling matrix element, and $\hbar$ is Planck's constant. A common classical expression for the Franck-Condon factor is ${ }^{22}$

$$
\mathrm{FC}=\frac{1}{\sqrt{4 \pi \lambda k_{B} T}} \exp \left(\frac{-(\lambda+\Delta G)^{2}}{4 \lambda k_{B} T}\right),
$$

where $\lambda$ is the reorganization energy, and $\Delta G$ is the free energy change of the reaction under the prevailing conditions of temperature, electrode-solution potential difference and environment.

Electron transfer at the semiconductor/liquid interface involves a continuum of electronic states in the semiconductor, whose solution, strictly speaking, requires solving a many-electronic state problem. A quantum mechanical study of the many-state crossing problem shows that when the splitting of the states caused by crossing is small the Landau-Zener formula is applicable to a large variety of such problems. ${ }^{23}$

The major charge carriers in these semiconductors have very low concentration and can be treated individually in interfacial reactions. ${ }^{24}$ As in the tight-binding calculations ${ }^{4}$ for the semiconductor/liquid interfacial electron transfer rate constant, it is assumed in the present study that only transitions between each pair of semiconductor/molecule states are important, and we restrict ourselves to this two-level approximation. Under this approximation the electron transfer current between the electrode and an acceptor state is the sum of the current from each electronic state of the semiconductor electrode to the molecular state, and a total rate constant (total denoted by t) $k_{f}^{\mathrm{t}}(\mathbf{r})$ can be written as $k_{f}^{\mathrm{t}}(\mathbf{r})$ $=\Sigma_{\mathbf{k}} k_{f}^{\mathrm{s}}(\mathbf{k}, \mathbf{r})$. Here, $\mathbf{k}$ denotes a semiconductor electronic state whose wave vector is $\mathbf{k}$. The $k_{f}^{\mathrm{t}}(\mathbf{r})$ varies with the position $\mathbf{r}$ of the acceptor molecule relative to the electrode, and can be further written as ${ }^{18}$

$$
k_{f}^{\mathrm{t}}(\mathbf{r})=\frac{2 \pi}{\hbar} \sum_{\mathbf{k}} \operatorname{FC}\left(\epsilon_{\mathbf{k}}\right) f\left(\epsilon_{\mathbf{k}}\right)\left|V_{\mathbf{k}}(\mathbf{r})\right|^{2},
$$

where $\epsilon_{\mathbf{k}}$ is the energy of the state $\mathbf{k}, f\left(\epsilon_{\mathbf{k}}\right)$ is the probability that the state $\mathbf{k}$ is occupied and $V_{\mathbf{k}}(\mathbf{r})$ is the coupling matrix element between the electronic state $\mathbf{k}$ of the semiconductor and the molecule. The FC and $\left|V_{\mathbf{k}}(\mathbf{r})\right|^{2}$ have units of energy $^{-1}$ and energy ${ }^{2}$, and $k_{f}^{\mathrm{t}}(\mathbf{r})$ has units of $\mathrm{s}^{-1}$. When $\epsilon_{\mathbf{k}}$ denotes the energy of state $\mathbf{k}$ relative to the edge of the conduction band, the $\Delta G$ in Eq. (8) is related to $\epsilon_{\mathbf{k}}$ by

$$
\Delta G=\Delta G^{0}-\epsilon_{\mathrm{k}},
$$

where $\Delta G^{0}$ is defined as the standard free energy of the reaction when the donor state in the semiconductor electrode is at the conduction band edge at the interface $\left(\epsilon_{\mathbf{k}}=0\right) . \Delta G^{0}$ can be obtained from electrochemical measurements.

An expression for the current density is given next in the terms of $k_{f}^{\mathrm{t}}(\mathbf{r})$. The forward current density through the electrode is obtained by first summing over currents from the electrode to all the acceptors in the solution and then dividing the sum by the area of the electrode surface $\sigma$,

$$
J_{f}=\frac{e}{\sigma} \int_{\mathbf{r}}[A(\mathbf{r})] k_{f}^{\mathrm{t}}(\mathbf{r}) d^{3} \mathbf{r} .
$$

When the reaction is not diffusion-controlled, and when the change of electrical potential inside the liquid can be neglected, as apparently it is under the condition in Lewis' experiments, ${ }^{1-3}[A(\mathbf{r})]$ can be taken as constant. The electron transfer rate constant in Eq. (4), which is independent of the concentration of acceptors in the solution but is implicitly dependent on the concentration of electrons in the semiconductor is then

$$
k_{f}=\frac{1}{\sigma} \int_{\mathbf{r}} k_{f}^{\mathrm{t}}(\mathbf{r}) d^{3} \mathbf{r} .
$$

It has units of $\mathrm{cm} \mathrm{s}^{-1}$. 
In particular, when $k_{f}(\mathbf{r})$ is only a function of $R$, the distance between the redox species and the electrode surface, Eq. (12) becomes

$$
k_{f}=\int_{R_{0}}^{\infty} k_{f}^{\mathrm{t}}(R) d R
$$

This equation, together with Eq. (9), will be used later in deriving an expression for the maximum electron transfer rate constant at a semiconductor/liquid interface.

\section{Electronic coupling matrix element}

In this section, the electronic coupling matrix element is obtained using the semiconductor and molecular electronic wave functions given in Appendices A and B as the zerothorder orbitals for the interacting system.

For an electron donor $(D)$ and acceptor $(A)$ system, if treated as a two-state problem, the coupling matrix element can be obtained by solving a secular equation $\operatorname{det}(\mathbf{H}-E \mathbf{S})$ $=0$, where $\mathbf{H}$ and $\mathbf{S}$ are the Hamiltonian and overlap matrices for the two-level system. When the two zeroth-order states have the same energy, or in the context of Eq. (14) below, $\langle D|H| D\rangle=\langle A|H| A\rangle$, the matrix element $T_{D A}$ is then half of the value of the difference between the two eigenvalues of the above secular problem, and can be expressed $\operatorname{as}^{25,26}$

$$
T_{D A}=\frac{\langle D|H| A\rangle-\langle D|H| D\rangle\langle D \mid A\rangle}{1-|\langle D \mid A\rangle|^{2}},
$$

where $\langle D \mid A\rangle$ is the electronic overlap integral of the donor and the acceptor state. The electron transfer between each semiconductor state and the molecular state will be treated as a two-state problem with the coupling matrix element obtained using Eq. (14). For a free electron model the Hamiltonian of such an interacting system is $H=-\hbar^{2} / 2 m \nabla^{2}+V$, where $V=V_{1}$ within the semiconductor, $V=V_{2}$ within the molecule, and $V=0$ everywhere else. In this case, the coupling matrix element denoted by $V_{\mathbf{k}}$ between the semiconductor state with wave vector $\mathbf{k}$ and the molecule, can be written as ${ }^{14,15,27,28}$

$$
\begin{aligned}
V_{\mathbf{k}} & =\frac{V_{1}\left\langle\psi \mid \Psi_{\mathbf{k}}\right\rangle_{1}-V_{1}\langle\psi \mid \psi\rangle_{1}\left\langle\psi \mid \Psi_{\mathbf{k}}\right\rangle}{1-\left|\left\langle\psi \mid \Psi_{\mathbf{k}}\right\rangle\right|^{2}} \\
& \cong V_{1}\left\langle\psi \mid \Psi_{\mathbf{k}}\right\rangle_{1}-V_{1}\langle\psi \mid \psi\rangle_{1}\left\langle\psi \mid \Psi_{\mathbf{k}}\right\rangle,
\end{aligned}
$$

where $\langle\cdots\rangle_{1}$ means the integration over the space occupied by the semiconductor. The term $\left|\left\langle\psi \mid \Psi_{\mathbf{k}}\right\rangle\right|^{2}$ in the denominator of the first equality can be neglected relative to unity as the volume of the semiconductor region becomes large.

To apply Bardeen's method to calculate $V_{\mathbf{k}}$, it is necessary to extend it to the present system where the electron mass $m_{2}$ in the molecule differs from the effective mass $m$ of the electron in the semiconductor. Further, the effective mass for an electron of the semiconductor has been defined only for the bulk properties, and yet an electron mass in the wave function just outside the semiconductor is needed also. With these observations in mind, we introduce the following procedure.

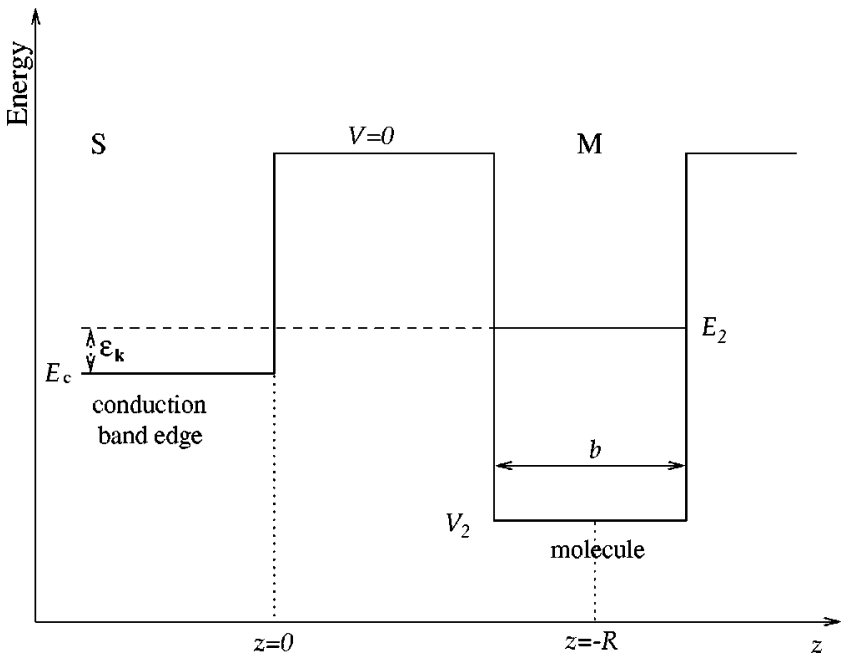

FIG. 1. Profile of potential wells of the semiconductor and of the spherical molecule. There is an electron tunneling through the intervening enviroment.

A typical fall-off factor of an electron transfer coupling matrix element with distance is $\exp (-\beta R / 2)$, where $R$ is distance from the relevant edge of one reactant to the relevant edge of the second reactant and $\beta \cong 1 \AA^{-1} .{ }^{22}$ We can achieve this distance dependence in the free electron model as follows: we write $R=R_{1}+R_{2}$, where $R_{1}$ is the distance from a field point along $\mathbf{R}$ to the edge of one reactant and $R_{2}$ is the distance from that point to the edge of the other reactant. In a free electron description each wave function then decreases as $\exp \left(-\beta R_{i} / 2\right), i=1,2$. For the semiconductor, denoting $\beta$ by $\beta_{1}$, we have $\beta_{1} / 2=\sqrt{-2 m\left(E_{c}+\epsilon_{\mathbf{k}}\right)} / \hbar$, where $E_{c}$ is the conduction band edge relative to the energy in the solvent, taken as zero. [The $E_{\mathbf{k}}$ given later in Eq. (16) is this $E_{c}+\epsilon_{\mathbf{k}}$.] Since $E_{c}$ is about $1-2 \mathrm{eV}$ and $\epsilon_{\mathbf{k}}$ is about $k_{B} T$, we have $\epsilon_{\mathbf{k}} \ll\left|E_{c}\right|$ and so $\beta_{1} / 2 \cong \sqrt{-2 m E_{c}} / \hbar$. For any choice for $m$, e.g., choosing it to equal to the effective mass in the bulk semiconductor, we can choose $E_{c}$ to yield the chosen $\beta_{1}\left(\sim 1 \AA^{-1}\right)$.

For the molecule the wave function and energy of the electron depends on the relevant molecular radius $b$, on the electron mass $m_{2}$, and on the position of the molecular energy level $E_{2}$ relative to the solvent, again taken as zero. Inasmuch as the relevant $\beta$, written as $\beta_{2}$, equals $2 \sqrt{-2 m_{2} E_{2}} / \hbar$, and we wish to have $\beta_{2} \cong \beta_{1}=\beta$. In the interests of simplicity, we can choose the pair $\left(m_{2}, E_{2}\right)$ so as to produce the desired $\beta_{2}$. If we take, for example, $m_{2}$ $=m$, the effective mass in the bulk semiconductor, we can adjust $E_{2}$ to achieve this $\beta_{2}$. The adjusted $E_{2}$ equals $E_{c}$ $+\epsilon_{\mathrm{k}}$ (and hence $\cong E_{c}$ ). Indeed, for electron transfer we have $E_{c}+\epsilon_{\mathrm{k}}=E_{2}$ in the transition state and so this selection of $m$ for the electron mass outside the molecule is consistent with this energy requirement (see Fig. 1).

It remains to consider the behavior of the molecular wave function inside the molecule. We have already fixed the energy $E_{2}$, a mass $m$, and a radius $b$. To achieve this $E_{2}$ for the given $b$ and $m$ we merely choose the appropriate depth of the potential energy well $V_{2}$. Accordingly, we now have a system which has the same electron mass $m$ through- 
out and yields the desired decrease of the wave function with distance.

The method due to Bardeen, ${ }^{16}$ used here for the evaluation of the value of $V_{\mathbf{k}}$, is only applicable when the donor and acceptor states have the same energy, which is the case for the electron transfer reaction obeying the FranckCondon principle and considered in this study. Since $\langle\psi \mid \psi\rangle_{1}\left\langle\psi \mid \Psi_{\mathbf{k}}\right\rangle$ is typically small as compared to $\left\langle\psi \mid \Psi_{\mathbf{k}}\right\rangle_{1},{ }^{29}$ the $V_{\mathbf{k}}$ in Eq. (15) can be approximated by $V_{1}\left\langle\psi \mid \Psi_{\mathbf{k}}\right\rangle_{1}$. Following Bardeen, ${ }^{16}$ this quantity can be written as an integral over the space occupied by one of the reactants, here the semiconductor $S$, we note that

$$
\left\langle\psi\left|T+V_{1}\right| \Psi_{\mathbf{k}}\right\rangle_{1}=E_{\mathbf{k}}\left\langle\psi \mid \Psi_{\mathbf{k}}\right\rangle_{1},
$$

where $E_{\mathbf{k}}$ is the eigenvalue corresponding to $\left|\Psi_{\mathbf{k}}\right\rangle$ and $T$ denotes the kinetic energy operator, $-\left(\hbar^{2} / 2 m\right) \nabla^{2}$, in coordinate space. But we also have $T|\psi\rangle=E_{2}|\psi\rangle$ in the region outside the molecule, where $E_{2}$ is the eigenvalue for the molecule. Thereby,

$$
\left\langle\Psi_{\mathbf{k}}^{*}|T| \psi^{*}\right\rangle_{1}=E_{2}\left\langle\Psi_{\mathbf{k}}^{*} \mid \psi^{*}\right\rangle_{1}=E_{2}\left\langle\psi \mid \Psi_{\mathbf{k}}\right\rangle_{1} .
$$

We have from Eqs. (16) and (17),

$$
\begin{aligned}
-\frac{\hbar^{2}}{2 m} \int_{1}\left(\psi^{*} \nabla^{2} \Psi_{\mathbf{k}}-\Psi_{\mathbf{k}} \nabla^{2} \psi^{*}\right) d^{3} \mathbf{r}= & \left(E_{\mathbf{k}}-E_{2}-V_{1}\right) \\
& \times\left\langle\psi \mid \Psi_{\mathbf{k}}\right\rangle_{1} .
\end{aligned}
$$

When $\langle D|H| D\rangle$ and $\langle A|H| A\rangle$ are set equal in the transition state, $E_{\mathbf{k}}$ and $E_{2}$ are not quite equal, but the difference between them ${ }^{30}$ is neglected. ${ }^{27,28} \mathrm{We}$ thus obtain, on neglecting the terms mentioned earlier,

$$
V_{\mathbf{k}}=-\frac{\hbar^{2}}{2 m} \int \mathbf{n} \cdot\left(\psi^{*} \nabla \Psi_{\mathbf{k}}-\Psi_{\mathbf{k}} \nabla \psi^{*}\right) d s,
$$

where $\mathbf{n}$ is a unit vector normal to the surface of well $S$ and pointing outward from $S$, i.e., in the direction of negative $z$, and $d s$ is the area element of the surface of well $S$. Setting $z=0$ at the semiconductor surface, Eq. (19) then becomes,

$$
V_{\mathbf{k}}=-\frac{\hbar^{2}}{2 m} \int_{z=0}\left\{\psi\left(\partial \Psi_{\mathbf{k}}^{*} / \partial z\right)-\Psi_{\mathbf{k}}^{*}(\partial \psi / \partial z)\right\} d s .
$$

In the following the implementation of Bardeen's method is illustrated by an evaluation of the electronic coupling matrix element between a semiconductor state and an $s$-type state of the molecular acceptor. The expressions used for $p_{z}$-like and $d_{z^{2}}$-like molecular wave functions are given in Appendix B. The integrand in Eq. (20) is evaluated at the semiconductor surface. We have

$$
\begin{aligned}
& \Psi_{\mathbf{k}}(x, y, z=0)=a_{1} e^{i\left(k_{x} x+k_{y} y\right)}, \\
& \partial \Psi_{\mathbf{k}} / \partial z(x, y, z=0)=\beta_{1} a_{1} e^{i\left(k_{x} x+k_{y} y\right)},
\end{aligned}
$$

and when an $s$-type orbital is used for the molecular acceptor, we have

$$
\psi(x, y, z=0)=\frac{2 A_{0}}{\sqrt{4 \pi}} \frac{e^{-\beta_{2}\left(\sqrt{\rho^{2}+R^{2}}-b\right) / 2}}{\beta_{2} \sqrt{\rho^{2}+R^{2}}},
$$

$\partial \psi / \partial z(x, y, z=0)$

$$
=-\frac{2 A_{0} R}{\sqrt{4 \pi}} \frac{1+\left(\beta_{2} / 2\right) \sqrt{\rho^{2}+R^{2}}}{\beta_{2}\left(\rho^{2}+R^{2}\right)^{3 / 2}} e^{-\beta_{2}\left(\sqrt{\rho^{2}+R^{2}}-b\right) / 2} .
$$

The normalization of $\Psi_{\mathbf{k}}$ will be made with respect to a large volume $v$, most of which encompasses the semiconductor surface. The $a_{1}$ in Eqs. (21) and (22) has units of $1 / \sqrt{v}$, and is given by Eq. (27) below, and the $A_{0}$ in Eqs. (23) and (24) has units of $\mathrm{cm}^{-1 / 2}$. A normalization to a delta function could have been introduced instead if we had introduced a $z$-dependent electric field inside the semiconductor similar to the actual field. However, the present procedure is simpler and should suffice for our purpose.

Equations (23) and (24) are obtained using Eq. (B7) and setting the coordinate of the center of the spherical potential well as $(0,0,-R), R$ being the distance between the center of the molecule and the semiconductor surface, $\rho$ being $\sqrt{x^{2}+y^{2}}$, and $d s$ being $2 \pi \rho d \rho$.

Equations (21)-(24) are next used for the evaluation of the coupling matrix element. For a semiconductor conduction band, the occupation of the electronic states is low enough to be considered as obeying Boltzmann statistics. Thus, only states within an energy range of $k_{B} T$ above the conduction band edge are important in the electron transfer reaction. Since $k=|\mathbf{k}|$ is only about $0.1 \AA^{-1}$ at room temperature, it is a good approximation to replace the term $e^{i\left(k_{x} x+k_{y} y\right)}$ by unity in Eqs. (21) and (22). A final expression for the coupling matrix element is then obtained by performing the integral in Eq. (20), yielding

$$
V_{\mathbf{k}}(R)=-A_{0} \frac{2 \sqrt{\pi} \hbar^{2}}{\beta_{2} m} a_{1}\left(1+\frac{1}{\beta_{2} R}\right) e^{-\beta_{2}(R-b) / 2} .
$$

In obtaining the above expression, the approximation that $\int_{u_{0}}^{\infty}\left(e^{-u} / u^{2}\right) d u \approx e^{-u_{0}} / u_{0}^{2}, \quad$ and $\int_{u_{0}}^{\infty}\left(e^{-u / u}\right) d u \approx e^{-u_{0} / u_{0}}$, when $u_{0} \gg 1$, are used. Here, $u=\left(\beta_{2} / 2\right) \sqrt{\rho^{2}+R^{2}}$, and $u_{0}$ $=\beta_{2} R / 2$, the value of $u$ at $\rho=0$. Because of $a_{1}, V_{\mathbf{k}}(R)$ is seen to be proportional to $1 / \sqrt{v}$.

The term $1 / \beta_{2} R$ is small compared to the other term in the parenthesis in Eq. (25) when $R$ is large. In the problems treated in this paper, $R$ is always greater than $4 \AA$, and the term $1 / \beta_{2} R$ can then be neglected. In this case, Eq. (25) becomes,

$$
V_{\mathbf{k}}(R)=-\sqrt{\pi} A_{0} a_{1} \frac{2 \hbar^{2}}{\beta_{2} m} e^{-\left(\beta_{2} / 2\right)(R-b)}, \quad(\psi=s),
$$

and so $V_{\mathbf{k}}(R)$ depends exponentially on the edge to edge distance $R-b$ between the semiconductor and the molecule. $A_{0}$ is given by Eq. (B8) or approximately by Eq. (B11).

The quantity $a_{1}$ in Eq. (26) is estimated as in Appendix A to be

$$
a_{1}=\sqrt{\frac{2}{v}} \frac{k_{z}}{\sqrt{k_{z}^{2}+\left(\beta_{1} / 2\right)^{2}}} \approx \sqrt{\frac{2}{v}} \frac{2 k_{z}}{\beta_{1}}
$$

the second equality arising because $k_{z} \ll \beta_{1}$.

Using the relation that $\beta_{1}=\beta_{2}$, and the above expression for $a_{1}$, the expression for $V_{\mathbf{k}}(R)$ then becomes 


$$
V_{\mathbf{k}}(R)=-\sqrt{\frac{2 \pi}{v}} A_{0} \frac{4 k_{z} \hbar^{2}}{\beta_{2}^{2} m} e^{-\beta_{2}(R-b) / 2}, \quad(\psi=s) .
$$

The procedure discussed above for the $s$-type orbital can also be applied to a system with other types of molecular orbitals. In the present study, when a $p_{z}$-like or $d_{z^{2}}$-like orbital is used for the molecular orbital, as it should be for the molecules considered here, the approximation that $1 / \beta_{2}$ $=1 / \beta_{1} \approx 1 \AA$ is also used. The wave function $\psi$ is given by Eqs. (B1) and (B2), with $l=1, m=0$ for a $p_{z}$-like orbital, and $l=2, m=0$ for a $d_{z^{2}}$-like orbital, and their normalization constants are given by Eqs. (B3)-(B5). The electronic coupling matrix elements for these orbitals are then calculated using Eq. (20).

\section{ESTIMATE OF THE MAXIMUM ELECTRON TRANSFER RATE CONSTANT}

We next obtain the expression for the maximum rate constant $k_{\mathrm{et}}^{\max }$ of the electron transfer reaction at a semiconductor/liquid interface, based on the free electron model given in the preceding section. We first discuss the $f\left(\epsilon_{\mathbf{k}}\right)$ term in Eq. (9) and then derive an expression for $k_{f}^{\mathrm{t}}$ using Eqs. (9) and (28).

For a low-doped semiconductor of the zincblende type, the occupation of its conduction band at the surface is low enough that the occupancy probability, $f\left(\epsilon_{\mathbf{k}}\right)$, of the state $\mathbf{k}$, the kinetic energy of which is $\hbar^{2} k^{2} / 2 m,{ }^{11}$ can be treated as obeying Boltzmann statistics. The sum $\Sigma_{\mathbf{k}} \cdots f\left(\epsilon_{\mathbf{k}}\right)$ in Eq. (9) can be written as an integral over $\mathbf{k}$-states, when properly normalized. The number of electrons in the semiconductor conduction band in the volume $v$ is $n_{s} v$, and the probability of finding one of these electrons in $d k_{x} d k_{y} d k_{z}$ is the Boltzmann factor $\exp \left(-\epsilon_{\mathrm{k}} / k_{B} T\right) d k_{x} d k_{y} d k_{z} /$ $\int_{-\infty}^{\infty} \int_{-\infty}^{\infty} \int_{-\infty}^{\infty} \exp \left(-\epsilon_{\mathbf{k}} / k_{B} T\right) d k_{x} d k_{y} d k_{z}$. When multiplied by $n_{s} v$ it becomes the probability that a state is occupied. The sum in Eq. (9) thus becomes

$$
\begin{aligned}
\sum_{\mathbf{k}} \operatorname{FC}\left(\epsilon_{\mathbf{k}}\right)\left|V_{\mathbf{k}}(\mathbf{r})\right|^{2} f\left(\epsilon_{\mathbf{k}}\right) \\
\quad=\frac{n_{s} v \iiint \mathrm{FC}\left(\epsilon_{\mathbf{k}}\right)\left|V_{\mathbf{k}}(\mathbf{r})\right|^{2} e^{-\epsilon_{\mathbf{k}} / k_{B} T} d k_{x} d k_{y} d k_{z}}{\iiint e^{-\epsilon_{\mathbf{k}} / k_{B} T} d k_{x} d k_{y} d k_{z}} .
\end{aligned}
$$

Since $\left|V_{\mathbf{k}}\right|^{2}$ is inversely proportional to $v$, the $v$ cancels. Equation (9) yields

$$
k_{f}^{t}(\mathbf{r})=n_{s} v \frac{2 \pi}{\hbar} \frac{\iiint \mathrm{FC}\left(\epsilon_{\mathbf{k}}\right)\left|V_{\mathbf{k}}(\mathbf{r})\right|^{2} e^{-\epsilon_{\mathbf{k}} / k_{B} T} d k_{x} d k_{y} d k_{z}}{\left(2 \pi m k_{B} T\right)^{3 / 2} / \hbar^{3}},
$$

where $V_{\mathbf{k}}$ for an $s$-like electron is given by Eq. (28), and for $p_{z}$-like and $d_{z^{2}}$-like electrons in the molecule is given by Eq. (B12) in Appendix B.

Integration over $k_{x}, k_{y}$, and $k_{z}$ is intermediately performed, and one obtains

$$
\begin{aligned}
k_{f}^{\mathrm{t}}(R)= & n_{s} \frac{2 \pi \hbar}{m\left(\beta_{2} / 2\right)^{4}} A_{0}^{2}\left(\frac{2 \lambda}{\lambda-\Delta G^{0}}\right)^{5 / 2} \sqrt{\frac{\pi k_{B} T}{\lambda}} \\
& \times e^{-\left[\left(\lambda+\Delta G^{0}\right)^{2} / 4 \lambda k_{B} T\right]} e^{-\beta_{2}(R-b)}, \quad \lambda-\Delta G^{0} \gg 0,
\end{aligned}
$$

where $\Delta G^{0}$ is the same as defined earlier and $\beta_{1} \approx \beta_{2}$ has been used. Equation (31) was obtained under the condition that $\lambda-\Delta G^{0} \gg 0$.

The $k_{f}^{\mathrm{t}}$ given by Eq. (31) is then introduced into Eq. (13) to yield an expression for $k_{f}$. The maximum electron transfer rate constant $k_{f}^{\max }$ is obtained by setting $\lambda+\Delta G^{0}=0$ in Eq. (31) to obtain

$k_{f}^{\max }=n_{s} \frac{\pi \hbar}{m\left(\beta_{2} / 2\right)^{5}} A_{0}^{2} \sqrt{\frac{\pi k_{B} T}{\lambda}} e^{-\beta_{2}\left(R_{0}-b\right)}, \quad(s$ electron $)$.

It is seen to be linearly dependent on $n_{s}$, the electron density near the surface of the semiconductor electrons. Here, $R_{0}$ is the smallest distance between the center of the molecule and the semiconductor surface. We then have an expression for the maximum second-order electron transfer rate constant written as

$$
k_{\mathrm{et}}^{\max }=\frac{32 \pi \hbar}{m \beta_{2}^{5}} A_{0}^{2} \sqrt{\frac{\pi k_{B} T}{\lambda}} e^{-\beta_{2}\left(R_{0}-b\right)}, \quad(s \text { electron }),
$$

where $A_{0}$ is given by Eqs. (B8) and (B9).

The above equation is then applied in the following to the two systems studied by Lewis and co-workers for a comparison with the experimental results. Following the discussion in the earlier section, $\beta_{2}$ is taken as $1 \AA^{-1}$.

The rate constant of the electron transfer reaction at the silicon/viologen ${ }^{2+/+}$ interface is estimated using Eq. (33) for a (hypothetical) $s$-like electron. At the $\mathrm{Si} /$ $N, N^{\prime}$-dimethyl-4,4'-bipyridylium ${ }^{2+}$ interface, one of the $\mathrm{Si} /$ viologen systems studied by Fajardo and Lewis, ${ }^{1,3}$ the radius of the spherical potential $b$ is estimated as $3 \AA{ }^{31}$ which gives approximately the size of the LUMO of the molecular acceptor. The $m$ was obtained from self-consistent band structure calculations to be $0.191 m_{e}{ }^{32}$ where $m_{e}$ is the mass of a free electron. Since the surface of the silicon semiconductor in the experiments is terminated by a single layer of hydrogen atoms to remove the dangling $\mathrm{Si}$ bonds, the value of $R_{0}$ is chosen as the value corresponding to the direct contact of the adsorbed hydrogen atoms and the acceptors and is about $5 \AA .^{33}$ The value of $\lambda$ obtained from a fit in Ref. 12 to the experimental data, ${ }^{1}$ is about $0.7 \mathrm{eV}$ and the calculated maximum rate constant is relatively insensitive to $\lambda$. When the maximum rate constant for this $s$-electron model is calculated using Eq. (33), the result in Table I is obtained, and compared there with the experimental results as well as the theoretical results obtained in Part I by the tight-binding method.

We turn next to the estimate of the electron transfer reaction rate constant at the $\mathrm{InP} / \mathrm{Me}_{2} \mathrm{Fc}^{+/ 0}$ interface. For this system, $b$ is taken as $0.6 \AA{ }^{34}$ the radius of a $\mathrm{Fe}^{2+}$, because of the localization of the LUMO at Fe atom. ${ }^{35}$ The (100) surface InP semiconductor used in the experiments is believed $^{36}$ to be terminated by a layer of oxygen atoms which saturate the dangling $\mathrm{P}$ bonds. The smallest distance $R_{0}$ between the center of the acceptor and the electrode is chosen to be $5 \AA$ which corresponds to the direct contact of the molecular acceptor (the whole ferrocene molecule) and the oxygen atom. ${ }^{33}$ The experimental effective mass $m$ of an 
TABLE I. Experimental and calculated maximum electron transfer rate constant. $^{\text {a }}$

\begin{tabular}{|c|c|c|c|c|c|}
\hline System & $\begin{array}{c}k_{\mathrm{et}}^{\max } \\
\text { (expt.) }\end{array}$ & $\begin{array}{c}k_{\mathrm{et}}^{\max } \\
(z \text {-trans. })^{\mathrm{d}}\end{array}$ & $\begin{array}{c}k_{\mathrm{et}}^{\max } \\
(\text { slab) }\end{array}$ & $\begin{array}{c}k_{\mathrm{et}}^{\max } \\
(\text { free } e)^{\mathrm{e}}\end{array}$ & $\begin{array}{c}k_{\mathrm{et}}^{\max } \\
\text { (free } e \text { ) }\end{array}$ \\
\hline $\mathrm{Si} /$ viologen ${ }^{2+/+}$ & $0.6^{\mathrm{b}}$ & 1.3 & 1.6 & $1.2\left(p_{z}\right)$ & $1.9($ ' s'”) \\
\hline $\mathrm{Si} / \mathrm{Me}_{2} \mathrm{Fc}^{+/ 0}$ & $\ldots$ & $\cdots$ & 0.17 & $0.024\left(d_{z^{2}}\right)$ & $1.2(" s, ")$ \\
\hline $\mathrm{InP} / \mathrm{Me}_{2} \mathrm{Fc}^{+/ 0}$ & $1-2^{\mathrm{c}}$ & 0.084 & 0.086 & $0.017\left(d_{z^{2}}\right)$ & $1.1(" s, ")$ \\
\hline
\end{tabular}

Units are $10^{-16} \mathrm{~cm}^{4} \mathrm{~s}^{-1}$.

${ }^{\mathrm{b}}$ From Ref. 12.

${ }^{c}$ From Ref. 10

${ }^{\mathrm{d}}$ From Ref. 4.

${ }^{\mathrm{e}}$ The result for viologen ${ }^{2+/+}$ was obtained using a $p_{z}$-like orbital and the result for $\mathrm{Me}_{2} \mathrm{Fc}^{+/ 0}$ was obtained using a $d_{z^{2}}$-like orbital. The results in the last column were obtained using a hypothetical $s$-like orbital and Eq. (33).

electron in the InP conduction band is $0.077 m_{e} \cdot{ }^{11,37}$ The reorganization energy $\lambda$ of the system is about $0.8 \mathrm{eV},{ }^{2}$ but $k_{\mathrm{et}}^{\max }$ is again relatively insensitive to $\lambda$. The estimated rate constant for the $s$-electron model is then given in Table I.

However, to compare with the real systems studied by Lewis and co-workers, ${ }^{1,2}$ a $p_{z}$-like orbital should be used for the viologen to be more consistent with the symmetry of the LUMO of the viologen ions. For the InP/Me ${ }_{2} \mathrm{Fc}^{+/ 0}$ system, since the LUMO of $\mathrm{Me}_{2} \mathrm{Fc}^{+}$has primarily $d_{z^{2}}$ character, ${ }^{35}$ the $d_{z^{2}}$-like orbital is used for the acceptor state of $\mathrm{Me}_{2} \mathrm{Fc}^{+}$. When a $p_{z^{-}}$or $d_{z^{2}}$-like orbital is used, we average the rate constant ${ }^{38}$ over the orientation of the orbital respect to the semiconductor surface, yielding the results in Table I.

For comparison, the maximum electron transfer rate constant at the $\mathrm{Si} / \mathrm{Me}_{2} \mathrm{Fc}^{+/ 0}$ interface is also calculated, although the data on the maximum rate constant for this system are absent. The effective electron mass for the $\mathrm{Si}$ conduction band is again taken as $0.191 m_{e}$, the reorganization energy is $0.8 \mathrm{eV}$, the radius of $\mathrm{Me}_{2} \mathrm{Fc}^{+/ 0}$ LUMO is $0.6 \AA$, and $R_{0}$ is taken as $4 \AA$. The results obtained using an $s$-type orbital and a $d_{z^{2}}$-like orbital are both given in Table I.

\section{DISCUSSION AND CONCLUSION}

\section{A. Discussion}

In the present paper, the free electron model is applied to the study of electron transfer reactions at semiconductor electrode/liquid interfaces. The electronic wave functions of the semiconductor are obtained in terms of plane waves in a semi-infinite potential well and the wave function of the acceptor is approximated to be a $p_{z}$-like or $d_{z^{2}}$-like orbital and for comparison results for an $s$-like orbital are also given, all for a spherical potential well. An analytic formula for the coupling matrix element is obtained for an $s$-like orbital using Bardeen's method, and then an expression for the electron transfer rate constant is obtained using this formula of the coupling matrix element.

The maximum electron transfer rate constants for $\mathrm{Si} /$ viologen ${ }^{2+/+}$ and $\mathrm{InP} / \mathrm{Me}_{2} \mathrm{Fc}^{+/ 0}$, the two systems studied experimentally by Lewis et al., are then estimated using Eq. (33). The maximum rate constants of both systems are compared with the experimental result, which is of order of $10^{-17}$ to $10^{-16} \mathrm{~cm}^{-4} \mathrm{~s}^{-1}$. The agreement is reasonable, considering the approximations involved, and the result is also in reasonable agreement with the theoretical results obtained in Part I using tight-binding calculations. As mentioned earlier, to mimic the experiments and the tight-binding calculations, a $p_{z}$-like orbital and a $d_{z^{2}}$-like orbital were used for the viologen ${ }^{2+/+}$ and $\mathrm{Me}_{2} \mathrm{Fc}^{+/ 0}$ ions, respectively, in the calculations of the coupling matrix elements for these two systems. The error is greater for $\mathrm{Me}_{2} \mathrm{Fc}^{+/ 0}$ ions, where the LUMO is assumed to be localized on the Fe atom. For comparison, an $s$-like orbital was also used for the calculation of the maximum rate constant. However, this $s$-like orbital is hypothetical, since the LUMO of these systems is not an $s$-orbital.

The difference between the theoretical maximum rate constants at $\mathrm{Si} /$ viologen ${ }^{2+/+}$ and $\mathrm{InP} / \mathrm{Me}_{2} \mathrm{Fc}^{+/ 0}$ interfaces in these calculations is partly due to the different size of the molecular orbitals. The LUMO, the electron acceptor state, of a viologen molecule is more delocalized than that of the $\mathrm{Me}_{2} \mathrm{Fc}$ molecule, which is essentially localized on the $\mathrm{Fe}$ atom. ${ }^{35}$ Although the centers of the two spherical potential wells representing the two molecules are at approximately the same distance from the semiconductor surface, the calculated electron transfer rate constant for the $\mathrm{Si} /$ viologen ${ }^{2+/+}$ interface is larger than that for the $\mathrm{InP} / \mathrm{Me}_{2} \mathrm{Fc}^{+/ 0}$. The coupling matrix element as a function of the size of the acceptor orbital is shown in Fig. 2. The distance between the center of the spherical orbital and the semiconductor surface is kept constant in obtaining this figure. Another factor responsible for the larger calculated maximum rate constant at the $\mathrm{Si} /$ viologen ${ }^{2+/+}$ interface is the character of the acceptor orbital. The use of a $p_{z}$-like orbital yields more efficient coupling between the semiconductor and the acceptor for the $\mathrm{Si} /$ viologen ${ }^{2+/+}$ interface than does the $d_{z^{2}}$-like orbital that used for the $\mathrm{InP} / \mathrm{Me}_{2} \mathrm{Fc}^{+/ 0}$ interface, both for the tightbinding and for the free electron calculations. The relative inefficiency of $d$-electron in electron transfer was described in an earlier paper. ${ }^{40}$

It is interesting that a model as simple as the free electron model yields a result for the electron transfer matrix element in reasonable agreement with the tight-binding calculation and with experiments. In these applications, the wave functions of the semiconductor or a reactant are needed outside the molecular potential well and on the surface of the semiconductor. For both wave functions boundary conditions are imposed (continuity of the respective wave function and of their derivatives at the relevant boundary). For the region outside the semiconductor and outside the molecule we introduce a distance dependence of the wave function which yielded the expected distance dependence of the electronic matrix element. Since the expected distance dependence is also reproduced quite well by extended-Hückel calculations, ${ }^{39}$ with no adjustable parameters, perhaps the agreement of the matrix element calculated using the free electron model with the obtained tight-binding/extendedHückel calculations or from experiments is also consistent with this earlier work. The analytical expression Eq. (28) serves to bring out some of the sources of error: when the molecule and the semiconductor are more or less in edge-toedge contact, as in the methyl viologen case, the exponential factor in Eq. (28) is of the order of unity, and so is not a major source of error. However, when the orbital in the mol- 


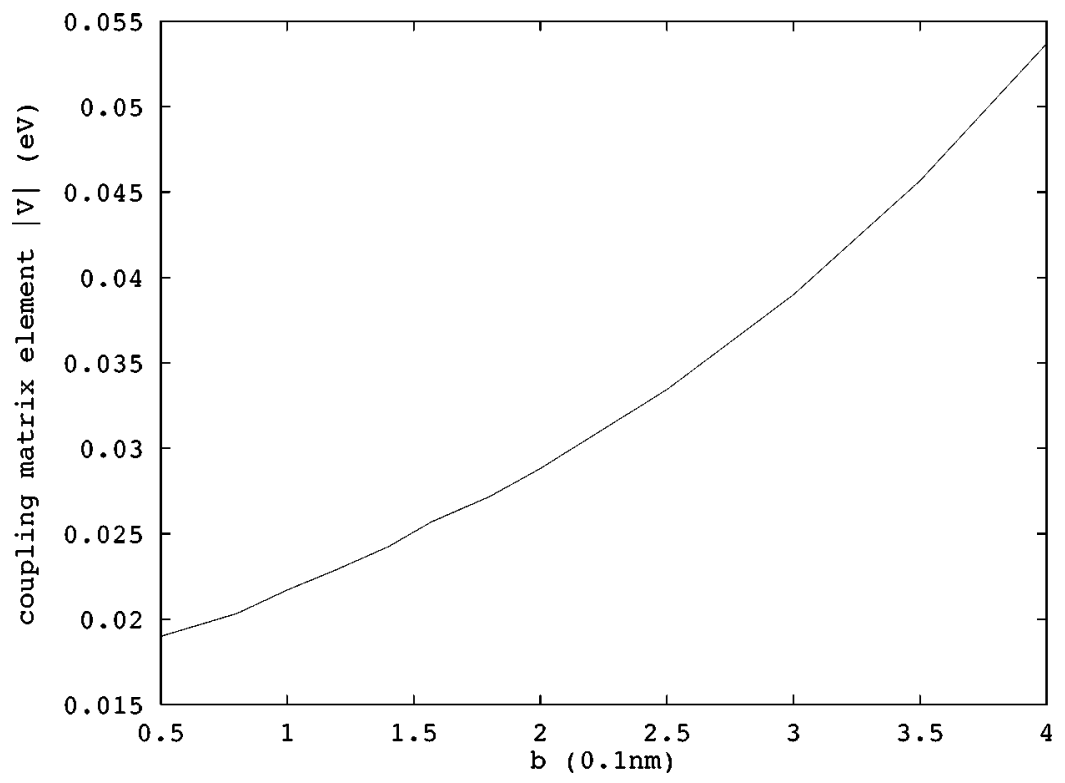

FIG. 2. The coupling matrix element between the semiconductor state $\mathbf{k}=(0,0,0.02)$ and an $s$-like molecular orbital as a function of the size of the acceptor state. The distance between the center of the molecule and the semiconductor surface is kept as a constant $5 \AA$.

ecule is localized, as in the ferrocene and so is buried, the orbital distance $R-b$, is large. Because of the exponential, the corresponding free electron model matrix element is subject to a substantially large error, as seen in Table I, using orbitals of the appropriate symmetry. Errors in the other quantities, e.g., for $A_{0}$ in Eq. (B11), appear to be more minor.

We explore further in the next section the relation between the free electron and the tight-binding models.

\section{B. Relation of free electron and tight-binding models}

We make this comparison initially for a one-dimensional chain of length $l$. For this chain the free electron value of $a_{1}$ is still given by Eq. (27), but with $\sqrt{v}$ replaced by $\sqrt{l}$. The tight-binding coefficients $C_{M}^{K}$ for a chain of $N$ atoms can be written as ${ }^{41}$

$$
C_{M}^{K}=\sqrt{2 /(N+1)} \sin \pi M K /(N+1),
$$

where $M$ is a lattice atom index $(M=1, \ldots, n), K$ is an electronic state index $(K=1, \ldots, N)$, and $M=1$ is a surface atom. Since the wave number $k_{z}=2 \pi / \lambda \cong \pi K /(N+1) a$, where $a$ is the lattice distance parameter, we can write

$$
C_{M}^{K}=\sqrt{2 a / l} \sin M k_{z} a .
$$

Inasmuch as the $C_{M}^{K}$, s are normalized to unity $\left(\Sigma_{M}\left|C_{M}^{K}\right|^{2}\right.$ $=1$ ) and the individual atomic wave functions are normalized over a length $a$, the $C_{M}^{K} / \sqrt{a}$ for $M=1$ is the quantity to compare with the one-dimensional analog of $a_{1}$ in Eq. (27), $\sqrt{2 / 2} 2 k_{z} / \beta_{1}$. Since $k_{z}$ is small, Eq. (35) yields $C_{1}^{K} / \sqrt{a}$ $\cong \sqrt{2 / l} k_{z} a$. When the tight-binding model is extended to the $x$ and $y$ directions, infinite in both directions, normalized to periodic boundary conditions (area $l^{2}$ ) using complex exponential wave functions, which are the discrete analogs of the $\exp i k_{x} x+i k_{y} y$ in Eq. (A2), Eq. (35) again applies but with $\sqrt{2 a / l}$ replaced by $\sqrt{2 a^{3} / v}$,

$$
C_{M}^{K}=\sqrt{2 a^{3} / v} \sin M k_{z} a .
$$

Omitted for brevity in the right-hand side of Eq. (36) are the discrete analogs of $\exp i k_{x} x+i k_{y} y$. For $M=1$ and with $k_{z} a$ being small, we can write

$$
a_{1} \cong 2 C_{1}^{K} / \beta_{1} a \sqrt{a^{3}},
$$

where $C_{1}^{K}$ is the same for each surface atom (for small $k_{x} x$ and $\left.k_{y} y\right)$. Thereby, $2 / \beta_{1} a \sqrt{a^{3}}$ can be regarded as the factor in $a_{1}$ contributing to the atom/atom exchange integral between the semiconductor and the adjacent solvent.

The contribution $C^{2}$ to $\int|\psi|^{2} 4 \pi r^{2} d r$ outside $r=b$ is, from Eq. (B7), $4 A_{0}^{2} / \beta_{2}^{3}$. Taking the coefficient $C$ of the molecule as unity, we can now rewrite $V_{\mathbf{k}}$ in Eq. (25) as

$$
V_{\mathbf{k}} \cong-\sqrt{\pi} \frac{4 A_{0} \hbar^{2}}{m \beta_{2} a^{5 / 2}} C C_{1}^{K},
$$

where $C \cong 1$. Now the lowest energy of an electron in a cubic box of edge length $a$ is $\epsilon=3 \hbar^{2} / 8 a^{2} m$. In terms of $\epsilon$, Eq. (38) becomes

$$
V_{\mathbf{k}}=-\sqrt{\frac{\pi}{\beta a}} \frac{4}{3 \pi^{2}} \epsilon C C_{1}^{K} .
$$

For a value of $\beta=1 \AA^{-1}, m=0.1 m_{e}$, and the lattice constant $a=3 \AA$, the factor multiplying $C C_{1}^{K}$ is about $2.5 \mathrm{eV}$. Not all of $C$ can contribute to $V_{\mathbf{k}}$, but more than one semiconductor atom, and its $C_{1}^{K}$, can contribute. To some extent these neglected aspects approximately cancel. The coefficient of $C C_{1}^{K}$ is seen to have (approximately) the value expected for an atom/atom exchange integral.

\section{Conclusion}

Although the free electron model is highly approximate, it does provide a reasonable description for the semiconductor electronic structure at the conduction band edge. ${ }^{11,32,42,43}$ The present model also incorporates the actual molecular orbital size and symmetry and the experimental coupling decay length, and perhaps for these reasons gives a reasonable zeroth-order approximation for treating the electron transfer 
at semiconductor/liquid interfaces. Although one cannot generalize from only two cases, we suspect on comparing the tight-binding and free electron results in Table I that the free electron model is better for a delocalized orbital like that of viologen than for a highly localized one like that of ferrocene. In this paper, for simplicity, the molecular orbital was first taken as an $s$-like orbital with a certain size, leading to an analytic result. However, the symmetry of the molecular orbital was taken into account instead by choosing orbitals with appropriate quantum numbers $l$ and $m$ in Eqs. (B6) and (B7).

In summary, it appears that the free electron model provides a reasonable and simple though crude description of the electron transfer reaction at semiconductor/liquid interfaces. Since this method uses Bardeen's method of estimating the coupling matrix element, it is not applicable to two overlapping potential wells ${ }^{16}$ and thus it is only applicable to relatively weak couplings. Also it is only applicable to the electron transfer reaction near the semiconductor conduction/ valence band edge, because of the use of the free electron model. This method of estimating the electron transfer rate constant can be readily applied to other semiconductor/liquid interfaces.

\section{ACKNOWLEDGMENTS}

It is a pleasure to acknowledge the support of the National Science Foundation and the Office of Naval Research. We thank Professor Nathan S. Lewis, William J. Royea, and Dr. Yuri Geogievskii for valuable discussions. We would like to dedicate this article to our esteemed colleague, Royce Murray, on the occasion of his forthcoming 65th birthday.

\section{APPENDIX A: FREE-ELECTRON MODEL FOR THE SEMICONDUCTOR ELECTRODE}

We treat the semiconductor electrode first and then the molecular electron acceptor in the solution using the potential wells. The electrons in the semiconductor are treated as free electrons in a potential well in the three-dimensional space, with a constant potential $V_{1}$ inside the well $\left(V_{1}\right.$ $<0)$. The potential well is infinite in the $x$ and $y$ directions and has a surface at $z=0$, and the potential is taken as zero outside the potential well. The wave functions are then obtained using the one-electron Schrödinger equation,

$$
-\frac{\hbar^{2}}{2 m} \nabla^{2}+V(\mathbf{r})\left|\Psi_{\mathbf{k}}\right\rangle=E_{\mathbf{k}}\left|\Psi_{\mathbf{k}}\right\rangle,
$$

where $V(\mathbf{r})=V_{1}$ when $z \geqslant 0$ and $V(\mathbf{r})=0$ when $z<0$. The $\mathbf{k}$ again denotes the wave vector of the electronic state $\Psi_{\mathbf{k}}$ and $m$ is the effective mass of the electron.

The relevant solution of Eq. (A1) is

$$
\Psi_{\mathbf{k}}(\mathbf{r})=e^{i\left(k_{x} x+k_{y} y\right)}\left(a_{2} e^{i k_{z} z}+a_{3} e^{-i k_{z} z}\right), \quad \text { (inside well), }
$$

for $z \geqslant 0$, and

$$
\Psi_{\mathbf{k}}(\mathbf{r})=a_{1} e^{i\left(k_{x} x+k_{y} y\right)} e^{\beta_{1} z / 2}, \quad(\text { outside well }),
$$

where $\beta_{1}$ is a positive number, for $z \leqslant 0$. Here, $k_{x}, k_{y}$, and $k_{z}(\beta)$ are the components of the wave vector $\mathbf{k}$ in $x, y$, and $z$ directions, respectively, with

$$
\frac{\hbar^{2}}{2 m}\left(k_{x}^{2}+k_{y}^{2}+k_{z}^{2}\right)=E-V_{1}, \quad \frac{\hbar^{2}}{2 m}\left(k_{x}^{2}+k_{y}^{2}-\beta_{1}^{2} / 4\right)=E,
$$

where $E$ is the energy of the electron.

In Eqs. (A2) and (A3), $a_{2}, a_{3}$, and $a_{1}$ are constants which can be obtained by satisfying the boundary condition at the surface of the semiconductor and by the normalization of the wave function. The boundary condition at $z \rightarrow \infty$ requires that $\beta_{1}>0$. The amplitude of $\Psi_{\mathbf{k}}^{*}(\mathbf{r})$ outside the potential well is considerably smaller than inside, a normalization of the wave function, $\left\langle\Psi_{\mathbf{k}} \mid \Psi_{\mathbf{k}}\right\rangle=1$, yields $\left|a_{2}\right|^{2}+\left|a_{3}\right|^{2}$ $=1 / v$ to a good approximation, where $v$ is the volume of the semiconductor as discussed in the text.

As usual, the relations between $a_{2}$ and $a_{3}$, and between $a_{2}$ and $a_{1}$ are obtained using the continuity of the wave function and its first derivative with respect to $z$ at $z=0$, and can be written as

$$
a_{3}=\frac{k_{z}-\mathrm{i} \beta_{1} / 2}{k_{z}+\mathrm{i} \beta_{1} / 2} a_{2}, \quad a_{1}=\frac{2 k_{z}}{k_{z}+\mathrm{i} \beta_{1} / 2} a_{2} .
$$

These two equalities combined with the normalization equation, $\left|a_{2}\right|^{2}+\left|a_{3}\right|^{2}=1 / v$, determine the three constants $a_{2}$, $a_{3}$, and $a_{1}$ up to an arbitrary phase factor. These quantities will be used later in calculate the coupling matrix element between the $\Psi_{\mathbf{k}}$ and the wave function of the molecule. From Eq. (A5) one can verify that $\left|a_{3}\right|^{2}=\left|a_{2}\right|^{2}$, and if, without loss of equality we choose $a_{1}$ to be real, then $a_{2}^{*}=a_{3}$ and Eq. (27) is obtained.

\section{APPENDIX B: THE ELECTRONIC WAVE FUNCTION OF THE ACCEPTOR MOLECULE}

For simplicity, we treat the electronic wave function of the molecule in liquid as an electron moving in a finite spherical potential well with a radius $b$. The potential $V_{2}(\mathbf{r})$ within the potential well is a constant $V_{2}$ and is zero outside. The problem is well known ${ }^{44}$ and the results will be used as follows.

The solution of the Schrödinger equation in the spherical polar coordinate $(r, \theta, \phi)$ gives the normalized wave functions which are continuous at $r=b,{ }^{14}$

$$
\begin{aligned}
\psi_{m l}(r, \theta, \phi ; E)= & A_{l} N_{m l} \Phi_{m}(\phi) P_{l}^{m}(\cos \theta) j_{l}(\alpha r) k_{l}\left(\beta_{2} b / 2\right) / \\
& j_{l}(\alpha b) e^{\beta_{2} b / 2} \quad \text { (inside well), }
\end{aligned}
$$

when $r \leqslant b$, and

$\psi_{m l}(r, \theta, \phi ; E)=A_{l} N_{m l} \Phi_{m}(\phi) P_{l}^{m}(\cos \theta) k_{l}\left(\beta_{2} r / 2\right) e^{\beta_{2} b / 2}$,

(outside well),

when $r \geqslant b$. For use in Eqs. (B1) and (B2) we have defined the $A_{l}$ 's In Eqs. (B3) and (B5) below by introducing there a factor $e^{-\beta_{2} b / 2}$, so as to make the $A_{l}$ 's less sensitive to $\beta_{2}$. Here, $m$ and $l$ are the usual quantum numbers, $\alpha$ $=\sqrt{2 m\left(E-V_{2}\right)} / \hbar, \quad \beta_{2} / 2=\sqrt{-2 m E} / \hbar, \quad \Phi_{m}(\phi) \quad$ is $e^{i m \phi} / \sqrt{2 \pi}, P_{l}^{m}$ is an associated Legendre polynomial, and $j_{l}$ 


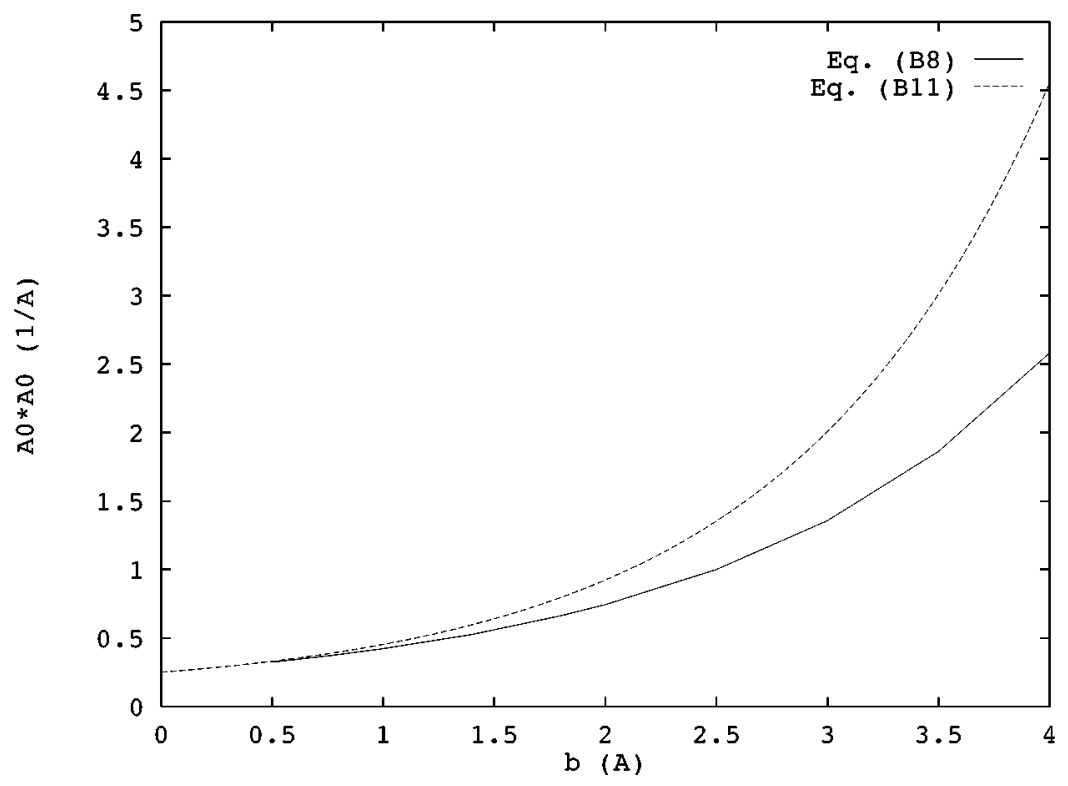

FIG. 3. The comparison for $A_{0}^{2}$ being calculated using Eq. (B8) (exact) and using Eq. (B11) (approximate).

and $k_{l}$ are spherical Bessel and Hankel functions. The normalization constants $A_{l}$ and $N_{m l}$ are given by

$$
\begin{aligned}
A_{l}= & \left\{\frac{k_{l}^{2}\left(\beta_{2} b / 2\right)}{j_{l}^{2}(\alpha b)} \int_{0}^{b} j_{l}^{2}(\alpha r) r^{2} d r\right. \\
& \left.+\int_{b}^{\infty} k_{l}^{2}\left(\beta_{2} r / 2\right) r^{2} d r\right\}^{-1 / 2} e^{-\beta_{2} b / 2},
\end{aligned}
$$

and

$$
N_{m l}=\left\{\frac{2 \pi}{2 l+1} \frac{(l+m) !}{(l-m) !} \nu\right\}^{-1 / 2} .
$$

$A_{l}$ can be further evaluated to be

$$
\begin{aligned}
A_{l}= & \left(2 / b^{3}\right)^{1 / 2}\left\{k_{l-1}\left(\beta_{2} b / 2\right) k_{l+1}\left(\beta_{2} b / 2\right)\right. \\
& \left.-\frac{k_{l}^{2}\left(\beta_{2} b / 2\right)}{j_{l}^{2}(\alpha b)} j_{l-1}(\alpha b) j_{l+1}(\alpha b)\right\}^{-1 / 2} e^{-\beta_{2} b / 2} .
\end{aligned}
$$

The $\nu$ in Eq. (B4) is 2 for $m=0$, and 1 for $m \neq 0$. In particular, $m=l=0$ corresponds to an $s$ state with a wave function denoted by $\psi$,

$$
\psi=\frac{2 A_{0}}{\sqrt{4 \pi}} \frac{\sin (\alpha r)}{\beta_{2} r \sin (\alpha b)}, \quad r \leqslant b
$$

and

$$
\psi=\frac{2 A_{0}}{\sqrt{4 \pi}} \frac{e^{-\beta_{2}(r-b) / 2}}{\beta_{2} r}, \quad r \geqslant b
$$

The constant $A_{0}$ obtained by setting $l=0$ in Eq. (B3) is given by

$$
A_{0}=\frac{\beta_{2}}{2}\left\{\frac{2 \alpha b-\sin (2 \alpha b)}{4 \alpha \sin ^{2}(\alpha b)}+\frac{1}{\beta_{2}}\right\}^{-1 / 2} .
$$

Clearly $A_{0}$ varies with the radius $b$ of the spherical potential well. The relation between $\alpha$ and $\beta_{2}$ in the last three equations, obtained by making $\partial \psi / \partial r$ continuous at $r=b$, gives

$$
\tan (\alpha b)=-2 \alpha / \beta_{2}
$$

For a given $\beta_{2}$ and $b$, the eigenvalue of the energy $E$ of the systems are then determined by the above equation and the relation between $\beta_{2}$ and $E$.

Using Eq. (B9), Eq. (B8) can be expressed as

$$
A_{0}=\frac{\beta_{2}}{2}\left\{\frac{\alpha^{2}+\left(\beta_{2} / 2\right)^{2}}{\alpha^{2}} \frac{2 \alpha b-\sin (2 \alpha b)}{4 \alpha}+\frac{1}{\beta_{2}}\right\}^{-1 / 2} .
$$

One notes from Eq. (B9) that $\tan (\alpha b)<0$, thus $2 \alpha b>\pi$ $>\sin (2 \alpha b)$. When $\alpha^{2}$ is sufficiently large, Eq. (B8) can be approximated by

$$
A_{0}=\frac{\left(\beta_{2}\right)^{3 / 2}}{2}\left[1 /\left(1+b \beta_{2} / 2\right)\right]^{1 / 2} .
$$

The $\alpha$ decreases monotonically when $b$ increases and is $0.73 \AA^{-1}$ when $b=3 \AA$. The $A_{0}^{2}$ calculated using Eqs. (B8) and (B11) are compared in Fig. 3. In the text and in Table I only Eq. (B8) is used. We note that $\int|\psi|^{2} 4 \pi r^{2} d r$ outside of the well of radius $b$ equals $4 A_{0}^{2} / \beta_{2}^{3}$, and we wish $4 A_{0}^{2} / \beta_{2}^{3}$ to be small. The value from Eq. (B11) is $\left[2 /\left(2+b \beta_{2}\right)\right]^{1 / 2}$.

As discussed in the text, the coupling matrix element between a molecular and a semiconductor state can be evaluated using

$$
V_{\mathbf{k}}=-\frac{\hbar^{2} a_{1}}{2 m} \int_{\rho=0}^{\infty} e^{i\left(k_{x} x+k_{y} y\right)}\left(\beta_{1} \psi_{m l}-\partial \psi_{m l} / \partial z\right) 2 \pi \rho d \rho,
$$


where the wave function $\psi_{m l}$ given by Eq. (B2) is a $p_{z}$-like orbital when $l=1, m=0$ and is a $d_{z^{2}}$-like orbital when $l$ $=2, m=0$, and where we have written the area element as $\rho d \rho d \phi$ and integrated the $\phi$ from 0 to $2 \pi$, noting that the integrand is independent of $\phi$.

${ }^{1}$ A. M. Fajardo and N. S. Lewis, J. Phys. Chem. B 101, 11136 (1997).

${ }^{2}$ K. E. Pomykal and N. S. Lewis, J. Phys. Chem. B 101, 2476 (1997).

${ }^{3}$ A. M. Fajardo and N. S. Lewis, Science 274, 969 (1996).

${ }^{4}$ Y. Q. Gao, Y. Georgievskii, and R. A. Marcus, J. Chem. Phys. 112, 3358 (2000).

${ }^{5}$ R. A. Marcus, J. Chem. Phys. 98, 5604 (1993).

${ }^{6}$ For example, S. G. Davison and J. D. Levine, Solid State Phys. 25, 1 (1970); D. J. Chadi and M. L. Cohen, Phys. Status Solidi B 68, 405 (1975); Y. R. Yang and C. B. Duke, Phys. Rev. B 36, 2763 (1987).

${ }^{7}$ B. Sapoval and C. Hermann, Physics of Semiconductors (Springer-Verlag, New York, 1993)

${ }^{8}$ S. M. Sze, Physics of Semiconductor Devices, 2nd ed. (Wiley, New York, 1981).

${ }^{9}$ B. K. Ridley, Quantum Processes in Semiconductors (Clarendon, Oxford, 1988).

${ }^{10}$ K. W. Böer, Survey of Semiconductor Physics (Van Nostrand Reinhold, New York, 1990).

${ }^{11}$ W. A. Harrison, Electronic Structure and the Properties of Solids (Dover, New York, 1989).

${ }^{12}$ C. Kittel, Introduction to Solid State Physics, 6th ed. (Wiley, New York, 1981), p. 193 or Fig. 9, p. 176. There it is seen that the curvature $d^{2} E / d k^{2}$, which is inversely proportional to the effective mass, is much larger at a band gap edge than is the curvature for a free electron. A k-p-based formula for the effective mass $m$ is derived in Ref. 11 and illustrates the factors influencing $m$.

${ }^{13}$ For example, J. R. Platt, K. Ruedenberg, C. W. Scherr, N. S. Ham, H. Labhart, and W. Lichten, Free-Electron Theory of Conjugated Molecules: A Source Book (Wiley, New York, 1964) (papers of the Chicago group 1949-1961), and references cited therein.

${ }^{14}$ P. Siders, R. J. Cave, and R. A. Marcus, J. Chem. Phys. 81, 5613 (1984).

${ }^{15}$ R. J. Cave, P. Siders, and R. A. Marcus, J. Phys. Chem. 90, 1436 (1986).

${ }^{16}$ J. Bardeen, Phys. Rev. Lett. 6, 57 (1961).

${ }^{17}$ W. Schmickler, J. Electroanal. Chem. 204, 31 (1986).

${ }^{18}$ R. R. Dogonadze and A. M. Kuznetsov, Prog. Surf. Sci. 6, 42 (1975).

${ }^{19}$ H. Ou-Yang, B. Källebring, and R. A. Marcus, J. Chem. Phys. 98, 7565 (1993).

${ }^{20}$ H. Ou-Yang, R. A. Marcus, and B. Källebring, J. Chem. Phys. 100, 7814 (1994).

${ }^{21}$ W. J. Royea, A. M. Fajardo, and N. S. Lewis, J. Phys. Chem. B 101, 11152 (1997).

${ }^{22}$ R. A. Marcus and N. Sutin, Biochim. Biophys. Acta 811, 265 (1985).

${ }^{23}$ Y. D. Demkov and V. I. Osherov, Sov. Phys. JETP 26, 916 (1968).

${ }^{24}$ H. Gerischer, J. Phys. Chem. 95, 1356 (1991).

${ }^{25}$ N. R. Kestner, J. Logan, and J. Jortner, J. Phys. Chem. 78, 2148 (1974).

${ }^{26}$ A. A. Stuchebrukhov, Chem. Phys. Lett. 265, 643 (1997).

${ }^{27}$ To obtain Eq. (15) from Eq. (14), we write the acceptor wave function as $\Psi_{\mathrm{k}}$, and the donor wave function as $\psi$. Using the Hamiltonian described in the text, we write it as $H=-\hbar / 2 m \nabla^{2}+V_{2} h_{2}+V_{1} h_{1}$, where $h_{1}$ is unity inside the semiconductor and zero everywhere else, while $h_{2}$ is unity inside the molecule and zero everywhere else. We can thus write $H$ as $H_{2}+V_{1} h_{1}$, where $H_{2}$ is the Hamiltonian for the molecule, $-\hbar^{2} / 2 m \nabla^{2}$ $+V_{2} h_{2}$. We then have $\left\langle\psi|H| \Psi_{\mathbf{k}}\right\rangle=\left\langle\psi\left|H_{2}\right| \Psi_{\mathbf{k}}\right\rangle+V_{1}\left\langle\psi\left|h_{1}\right| \Psi_{\mathbf{k}}\right\rangle$ $=E_{2}\left\langle\psi \mid \Psi_{\mathbf{k}}\right\rangle+V_{1}\left\langle\psi \mid \Psi_{\mathbf{k}}\right\rangle_{1}$, the 1 subscript indicating that the integration is over the volume of the semiconductor, and $E_{2}$ denoting the eigenvalue for the molecule, $\left\langle\psi\left|H_{2}\right| \Psi_{\mathbf{k}}\right\rangle=E_{2}\left\langle\psi \mid \Psi_{\mathbf{k}}\right\rangle$. Further, $\langle\psi|H| \psi\rangle=\langle\psi| H_{2}$ $+h_{1} V_{1}|\psi\rangle=E_{2}+V_{1}\langle\psi \mid \psi\rangle_{1}$, since $\langle\psi \mid \psi\rangle=1$. Thus the numerator in the right-hand side of Eq. (14) becomes that of Eq. (15).

${ }^{28}$ A. A. Stuchebrukhov, J. Chem. Phys. 105, 10819 (1996), which also, in effect, extends Bardeen's method.
${ }^{29}$ The calculated $\langle\psi \mid \psi\rangle_{1}\left\langle\psi \mid \Psi_{\mathbf{k}}\right\rangle /\left\langle\psi \mid \Psi_{\mathbf{k}}\right\rangle_{1}$ for $s, p$, and $d$ orbitals are less than 0.007 for the cases considered in this paper.

${ }^{30} \mathrm{We}$ note that $E_{\mathbf{k}}$ and $E_{2}$ are related as follows $E_{2}=\left\langle\psi\left|T+h_{2} V_{2}\right| \psi\right\rangle$ $=\langle\psi|H| \psi\rangle-\left\langle\psi\left|h_{1} V_{1}\right| \psi\right\rangle$ and the last term equals $-V_{1}\langle\psi \mid \psi\rangle_{1}$. Further, $E_{\mathbf{k}}=\left\langle\Psi_{\mathbf{k}}\left|T+h_{1} V_{1}\right| \Psi_{\mathbf{k}}\right\rangle=\left\langle\Psi_{\mathbf{k}}|H| \Psi_{\mathbf{k}}\right\rangle-\left\langle\Psi_{\mathbf{k}}\left|h_{2} V_{2}\right| \Psi_{\mathbf{k}}\right\rangle$ and the last term equals $-V_{2}\left\langle\Psi_{\mathbf{k}} \mid \Psi_{\mathbf{k}}\right\rangle_{2}$, the 2 indicating that it is integrated over the volume of the molecule. Since $\langle\psi|H| \psi\rangle=\left\langle\Psi_{\mathbf{k}}|H| \Psi_{\mathbf{k}}\right\rangle$, it then follows that $E_{\mathbf{k}}-E_{2}=-V_{2}\left\langle\Psi_{\mathbf{k}} \mid \Psi_{\mathbf{k}}\right\rangle_{2}+V_{1}\langle\psi \mid \psi\rangle_{1}$. However, terms such as $\langle\psi \mid \psi\rangle_{1}$ and $\left\langle\Psi_{\mathbf{k}} \mid \Psi_{\mathbf{k}}\right\rangle_{2}$ are neglected, tacitly or explicitly (Refs. 27,28).

${ }^{31}$ The LUMO of the viologen ${ }^{2+}$ ion is obtained by an extended Hückel calculation using M. H. Whangbo et al., Extended Hückel Molecular, Crystal and Properties Package, QCPE Program No. 571.

${ }^{32}$ The minima of $E(\mathbf{k})$ for Si shows ellipticity and two different effective masses can be distinguished, parallel and orthogonal to the axis (100). Since we are interested in the electron transfer across the (100) surface, the mass parallel to the (100) axis is used.

${ }^{33}$ Because of the asymmetry of the molecules studied in the experiments, there is some arbitrariness in estimating their radii and their closest distance to the semiconductor surface. This could bring in some error in estimating the electron transfer rate constant. The van der Waals' radii used in this study are taken from D. Freifelder, Physical Chemistry for Students of Biology and Chemistry (Science Books International, Boston, 1982), p. 60 : $\mathrm{H}(1.2 \AA), \mathrm{O}(1.4 \AA)$, and $\mathrm{C}(1.5 \AA)$. The $\mathrm{SiH}(1.55 \AA)$ and PO $(1.84 \AA)$ bond lengths are taken from D. F. Shriver, P. W. Atkins, and C. H. Langford, Inorganic Chemistry (Freeman, New York, 1990), p. 68.

${ }^{34}$ R. D. Shannon and C. T. Prewitt, Acta Crystallogr., Sect. B: Struct. Crystallogr. Cryst. Chem. 25, 925 (1969).

${ }^{35}$ G. L. Miessler and D. A. Tarr, Inorganic Chemistry (Prentice-Hall, New Jersey, 1999); A. Haaland, Acc. Chem. Res. 12, 415 (1979); J. Giordan, J. H. Moore, and J. A. Tossel, ibid. 19, 281 (1979); E. Rühl and A. P. Hitchcock, J. Am. Chem. Soc. 111, 5069 (1989)

${ }^{36} \mathrm{~N}$. Lewis group (private communication).

${ }^{37}$ W. J. Turner, W. E. Reese, and G. D. Pettit, Phys. Rev. 136, 157 (1964).

${ }^{38}$ When a $p_{z}$-like orbital $(l=1, m=0)$ or a $d_{z^{2}}$-like orbital $(l=2, m=0)$ is used, the coupling matrix element between the molecule and the semiconductor depends on the orientation of the orbital with respect to the semiconductor surface. Denoting the angle between the $z$ axis, which is normal to the semiconductor surface, and the body fixed $z$ axis of the molecule, which is along a maximum of the orbital, $\alpha$, the angular function of the oriented orbital can be written as $P_{l}^{0}[\cos \theta \cos \alpha+\sin \theta \sin \alpha]$ $=P_{l}^{0}(\cos \theta) P_{l}^{0}(\cos \alpha)+2 \Sigma_{m=1}^{l}[(l-m) ! /(l+m) !] P_{l}^{m}(\cos \theta) P_{l}^{m}(\cos \alpha) ; \quad[\mathrm{H}$. Hochstadt, Special Functions of Mathematical Physics (Holt, Rinehart, and Wintson, New York, 1961), p. 35.] Here, $P_{l}^{m}$ again denotes the Legendre polynomial. Because of symmetry, the contribution of the terms $P_{l}^{m}$ with $l=1, m=1$ or $l=2, m=1,2$ to the coupling between the oriented orbital and the semiconductor vanishes. The coupling between an oriented orbital and the semiconductor can be expressed as $P_{l}^{0}(\cos \alpha)\left\langle\psi_{0 l}|H| \Psi_{\mathbf{k}}\right\rangle$. The $\left\langle\psi_{0 l}|H| \Psi_{\mathbf{k}}\right\rangle$ denotes the coupling matrix element between the wave function $\psi_{0 l}$, defined by Eq. (B1), and the semiconductor wave function $\Psi_{\mathbf{k}}$ and can be evaluated using Bardeen's method described in Sec. II C. The average for the rate constant over the angle $\alpha$ then yields a factor $\int_{0}^{\pi}\left(P_{1}^{0}(\cos \theta)\right)^{2} d \alpha / \pi=\int_{0}^{\pi} \cos ^{2} \alpha d \alpha / \pi=0.5$ for a $p_{z}$-like orbital, and a factor $\int_{0}^{\pi}\left(P_{2}^{0}(\cos \alpha)\right)^{2} d \alpha / \pi=\left(\int_{0}^{\pi}\left(3 \cos ^{2} \alpha-1\right)^{2} / 4 d \alpha\right) / \pi=0.34$ for a $d_{z^{2}}$-like orbital.

${ }^{39}$ P. Siddarth and R. A. Marcus, J. Phys. Chem. 94, 2985 (1990).

${ }^{40}$ S. Gosavi and R. A. Marcus, J. Phys. Chem. B 104, 2067 (2000).

${ }^{41}$ For example, T. A. Hoffman and A. Konya, Acta Phys. Acad. Sci. Hung. 1, 5 (1951), Eq. (5.16).

${ }^{42}$ J. Bardeen, J. Chem. Phys. 6, 367 (1938).

${ }^{43}$ F. Bechstedt and R. Enderlein, Semiconductor Surfaces and Interfaces (Akademie-Verlag, Berlin, 1988).

${ }^{44}$ For example, L. I. Schiff, Quantum Mechanics, 3rd ed. (McGraw-Hill, New York, 1968). 